УДК 544.473

СВЯЗЬ СЕЛЕКТИВНОСТИ И СТРУКТУРНЫХ ФАКТОРОВ

ИНТЕРКАЛИРОВАННЫХ МОНТМОРИЛЛОНИТОВ

В РЕАКЦИИ ФИШЕРА-ТРОПША

\title{
RELATION BETWEEN SELECTIVITY AND STRUCTURE FACTORS OF INTERCOLLATED MONTMORILLOMITES IN FISCHER-TROPSCH REACTION
}

Давлетшин А.Р., Хамзин Ю.А., Шириязданов Р.Р., Пасько П.А., Вакулин И.В., Талипов Р.Ф.

Уфимский государственный нефтяной технический университет, г. Уфа, Российская Федерация

Башкирский государственный университет,

Уфа, Российская Федерация

\author{
A.R. Davletshin, Yu.A. Khamzin, R.R. Shiriyazdanov, P.A. Pasko, \\ I.V.Vakulin, R.F. Talipov \\ Ufa State Petroleum Technological University, \\ Ufa, Russian Federation \\ Bashkir State University, Ufa, Russian Federation
}

Аннотация. Процесс интеркалирования слоистых силикатов, например таких как монтмориллонит, путем внедрения в межслоевое пространство полиоксикатионов, образующихся в результате реакции гидролиза катионов, является одним из перспективных способов увеличения каталитической активности слоистых силикатов. Действительно, монтмориллонит, характеризующийся в естественном состоянии расширяющейся структурной ячейкой и толщиной силикатного слоя 0,94 нм, недостаточно активен. Однако в процессе интеркалирования 
происходит увеличение межслоевого пространства вследствие замещения межслоевых катионов на крупные олигомерные катионы и при этом активность монтмориллонита возрастает. Важно отметить, что процесс интеркалирования не приводит к нарушению первичной структуры слоистых силикатов. Ранее авторами были синтезированы катализаторы синтеза Фишера-Тропша на основе гетерополикислот, нанесенных на нанокомпозитный кислотно-активированный монтмориллонит. В настоящей работе представлены результаты моделирования процессов образования целевых продуктов методом молекулярной динамики, реализованным в модуле Adsorption Locator программного пакета Accelrys Material Studio 6.0. Влияние размеров полости на селективность образования изоалканов $\mathrm{C}_{6}-\mathrm{C}_{12}$ оценивалось по зависимости энергии адсорбции от размеров пор. Расчет структур олигомеров осуществлялся методом квантово-химического моделирования в приближении RM1.

Abstract. The intercalation process of layered silicates, for example, such as montmorillonite, by introducing into the interlayer space the polyoxycations produced by the cation hydrolysis reaction, is one of the promising ways to increase the catalytic activity of layered silicates. Indeed, montmorillonite, characterized in its natural state by the expanding structural cell and thickness of the silicate layer $0.94 \mathrm{~nm}$, is not sufficiently active. However, during intercalation, the interlayer space increases due to the substitution of interlayeric cations for large oligomeric cations, and the activity of montmorillonite increases. It is important to note that the intercalation process does not lead to a violation of the primary structure of layered silicates. Previously, the authors synthesized Fischer-Tropsch synthesis catalysts based on heteropolyacids applied to nanocomposite acid-activated montmorillonite. In this paper we present the results of modeling the processes of formation of the target products by the molecular dynamics method implemented in the Adsorption Locator module of Accelrys Material Studio 6.0. The effect of the cavity dimensions on the selectivity of the formation of $\mathrm{C}_{6}-\mathrm{C}_{12}$ isoalkanes was estimated from the 
dependence of the adsorption energy on the pore sizes. Calculation of oligomer structures was carried out by the method of quantum-chemical modeling in the RM1 approximation

Ключевые слова: синтез Фишера-Тропша, монтмориллонит, интеркаляция, энергия адсорбции, молекулярная динамика.

Key words: Fischer-Tropsch synthesis, montmorillonite, intercalation, adsorption energy, molecular dynamics.

\section{Введение}

Наиболее распространенными гетерогенными катализаторами синтеза Фишера-Тропша являются катализаторы, содержащие металлы VIII группы - Ni, Co, Fe с добавлением оксидов других металлов (Ti, Th, Mg, $\mathrm{Zr}$ ). В качестве носителей катализаторов, как правило, применяют различные оксиды, цеолиты, природные и синтетические алюмосиликаты $[1,2]$. Изменение структурных характеристик аморфных силикатов увеличение межслоевого пространства посредством интеркалирования поливалентыми катионами - напрямую влияет на каталитические свойства катализатора и селективность образования целевых углеводородов.

Исходный монтмориллонит (MON) представляет собой слоистый минерал с расширяющейся структурой следующей упрощенной формулы: $\mathrm{Al}_{4}\left[\mathrm{Si}_{8} \mathrm{O}_{20}\right](\mathrm{OH})_{4} \cdot n \mathrm{H}_{2} \mathrm{O}$. Пилларированный монтмориллонит относится к столбчатым глинам, преимуществом которых является не только сохранение большого числа доступных центров, но и появление новых пор. Возникает молекулярно-ситовая двухмерная структура с прямоугольными порами размерами до 2 нм, то есть размеры пор больше, чем у цеолитов, и приближаются к размерам мезопор.

Ранее авторами были синтезированы бифункциональные катализаторы синтеза Фишера-Тропша на основе модифицированного монтмориллонита. 
Химический состав исследуемого образца катализатора: $\mathrm{ZrO}_{2}-19,10 \%$ мacc., $\mathrm{SO}_{4}{ }^{2-}-15,30 \%$ мacc., $\mathrm{Co}-5,60 \%$ мacc., $\mathrm{ZrRu}-\mathrm{R}-0,25 \%$ мacc.

При этом показано, что в результате интеркаляции состав продуктов олигомеризации изменяется специфическим образом, в частности возрастает доля углеводородов изостроения $\mathrm{C}_{6}-\mathrm{C}_{12}$.

\section{Экспериментальная часть}

Предметом данного исследования стало изучение влияния особенностей микроструктуры модифицированных образцов MON на селективность образования углеводородов изостроения $\mathrm{C}_{6}-\mathrm{C}_{12}$ в процессе синтеза Фишера-Тропша. Для этого с использованием современного подхода «product shape selectivity» [3-5] была проведена оценка влияния интеркаляции ионов в монтмориллонит на состав продуктов синтеза Фишера-Тропша за счет изменения размеров межслоевого пространства в MON. В качестве количественного критерия использовалась расчетная энергия адсорбции продуктов в зависимости от размеров полости.

Использование альтернативных подходов «transition state shape selectivity» [6, 7] и «reagent shape selectivity» [8] в данном случае затруднено, т.к. неизвестны переходное состояние и состав промежуточных продуктов, а базовые реагенты для всех продуктов олигомеризации имеют одинаковое строение.

$\mathrm{B}$ качестве модельных продуктов рассмотрены изоалкены $\mathrm{C}_{6}, \mathrm{C}_{8}, \mathrm{C}_{9}$ и $\mathrm{C}_{12}$, поскольку являются наиболее предпочтительными в процессе синтеза Фишера-Тропша, а также ими лимитируется состав целевого продукта как компонента товарного автобензина (таблица 1).

Строение модельных продуктов выбрано исходя из пространственногеометрических характеристик и возможности определения предпочтительного направления образования продуктов. 
Таблица 1. Модельные продукты реакции синтеза Фишера-Тропша

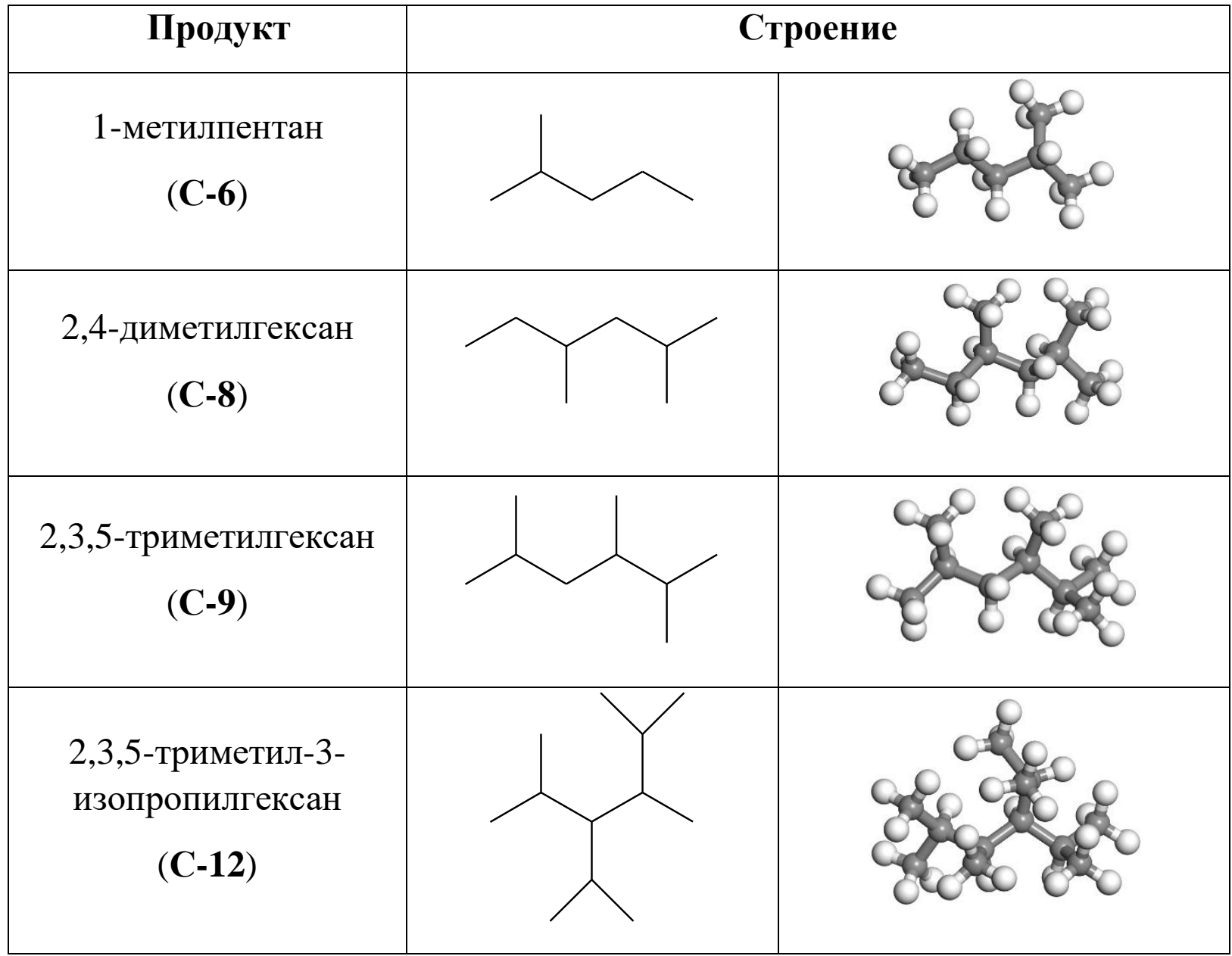

Моделирование процессов адсорбции и вычисление энергий осуществлялось с помощью метода молекулярной динамики, реализованного в модуле Adsorption Locator программного пакета Accelrys Material Studio 6.0 [9].

В качестве модельных структур выбраны пористые материалы с размерами пор от 8 до $12 \AA$, наиболее близкими к структуре катализатора, являющегося предметом исследования [5-11].

\section{Обсуждение экспериментальных данных}

Ниже представлена зависимость расчетных значений энергии адсорбции использованных углеводородов $\mathrm{C}_{6}-\mathrm{C}_{12}$ на модельных структурах в зависимости от их диаметра (таблица 2, рисунок 1). 
Таблица 2. Энергия адсорбции $\mathrm{E}_{\text {адс }}$ Углеводородов $\mathrm{C}_{6}-\mathrm{C}_{12}$ на рассмотренных моделях

\begin{tabular}{|c|c|c|c|c|c|}
\hline \multirow{2}{*}{$\begin{array}{c}\text { Модельная } \\
\text { структура }\end{array}$} & \multirow{2}{*}{$\mathrm{d}, \AA$} & \multicolumn{4}{|c|}{$\mathrm{E}_{\text {адс }}$, кДж/моль } \\
\cline { 3 - 6 } & & $\mathrm{C}-6$ & $\mathrm{C}-8$ & $\mathrm{C}-9$ & $\mathrm{C}-12$ \\
\hline BOG & 8,05 & $-131,1266$ & $-155,2034$ & $-170,2932$ & $-214,1832$ \\
\hline DON & 8,79 & $-112,1494$ & $-143,2486$ & $-155,3288$ & $-191,6948$ \\
\hline BPH & 9,51 & $-88,3234$ & $-105,3360$ & $-114,4902$ & $-133,5928$ \\
\hline LTN & 10,13 & $-120,7184$ & $-148,7662$ & $-160,3866$ & $-203,8168$ \\
\hline LTA & 11,05 & $-112,1076$ & $-141,7020$ & $-152,8208$ & $-196,0838$ \\
\hline SBE & 12,54 & $-116,1622$ & $-128,8276$ & $-147,1778$ & $-180,1580$ \\
\hline
\end{tabular}

Экстремальный характер представленных зависимостей наблюдается для всех углеводородов изостроения $\mathrm{C}_{6}-\mathrm{C}_{12}$ и свидетельствует о наименьшем сродстве модельной структуры с диаметром полости в районе 9,51 $\AA$ к данным продуктам процесса синтеза Фишера-Тропша, что отражается в минимальной энергии адсорбции (рисунок 1). Согласно идее «product shape selectivity» [10], малое сродство каталитической системы к продукту приводит к легкому удалению молекул из зоны реакции в канале матрицы и исключению их из дальнейших превращений. В случае синтеза Фишера-Тропша это приводит к увеличению выхода целевых углеводородов изоалканов $\mathrm{C}_{6}-\mathrm{C}_{12}$.

Таким образом, одним из факторов, влияющих на селективность образующихся изоалканов в ходе синтеза Фишера-Тропша на катализаторах, полученных в результате комплекса модифицирования монтмориллонита, является диаметр поры. Очевидно, что размером последнего можно управлять, изменяя диаметр интеркалируемых ионов и применяя различные приемы модификации. 


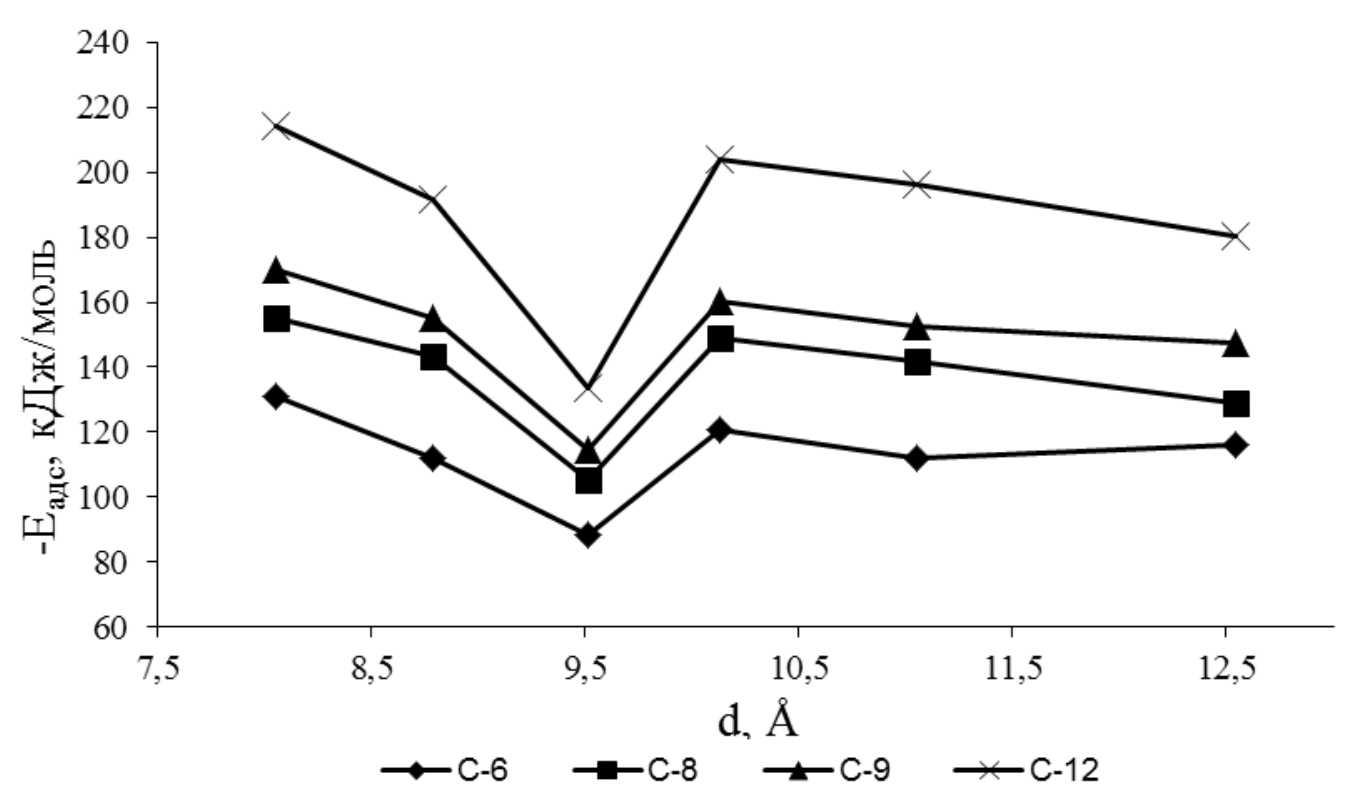

Рисунок 1. Зависимость энергии адсорбции углеводородов $\mathrm{C}_{6}$ и $\mathrm{C}_{12}$ от диаметра пор

С целью оценки влияния элементного состава полости и полярности внутриполостной поверхности рассмотрена зависимость энергии адсорбции изоалканов $\mathrm{C}_{6}-\mathrm{C}_{12}$ на углеродных нанотрубках (CNT), неполярных по своей природе.

Зависимости расчетных значений энергии адсорбции использованных углеводородов изостроения $\mathrm{C}_{6}-\mathrm{C}_{12}$ от диаметров $\mathrm{CNT}$ представлены в таблице 3 и на рисунке 2.

Таблица 3. Энергия адсорбции $\mathrm{E}_{\text {адс }}$ углеводородов $\mathrm{C}_{6}-\mathrm{C}_{12}$ на $\mathrm{CNT}$ различных диаметров

\begin{tabular}{|c|c|c|c|c|}
\hline \multirow{2}{*}{$\mathrm{d} \mathrm{CNT}, \AA$} & \multicolumn{4}{|c|}{ Энергия адсорбции $\mathrm{E}_{\text {адс, }}$ кДж/моль } \\
\cline { 2 - 5 } & $\mathrm{C}-6$ & $\mathrm{C}-8$ & $\mathrm{C}-9$ & $\mathrm{C}-12$ \\
\hline 8,14 & $-48,1118$ & $-62,6582$ & $-65,1662$ & $-69,2208$ \\
\hline 8,82 & $-52,8352$ & $-65,5842$ & $-67,8414$ & $-72,1886$ \\
\hline 9,52 & $-173,9298$ & $-227,1830$ & $-224,9258$ & $-72,3140$ \\
\hline 10,18 & $-155,4542$ & $-188,2254$ & $-230,0672$ & $-73,6516$ \\
\hline 10,88 & $-139,3612$ & $-182,2480$ & $-202,3538$ & $-249,9222$ \\
\hline 11,52 & $-128,7022$ & $-166,4476$ & $-182,4152$ & $-235,8774$ \\
\hline 12,23 & $-119,8824$ & $-154,1166$ & $-166,7402$ & $-209,7942$ \\
\hline
\end{tabular}




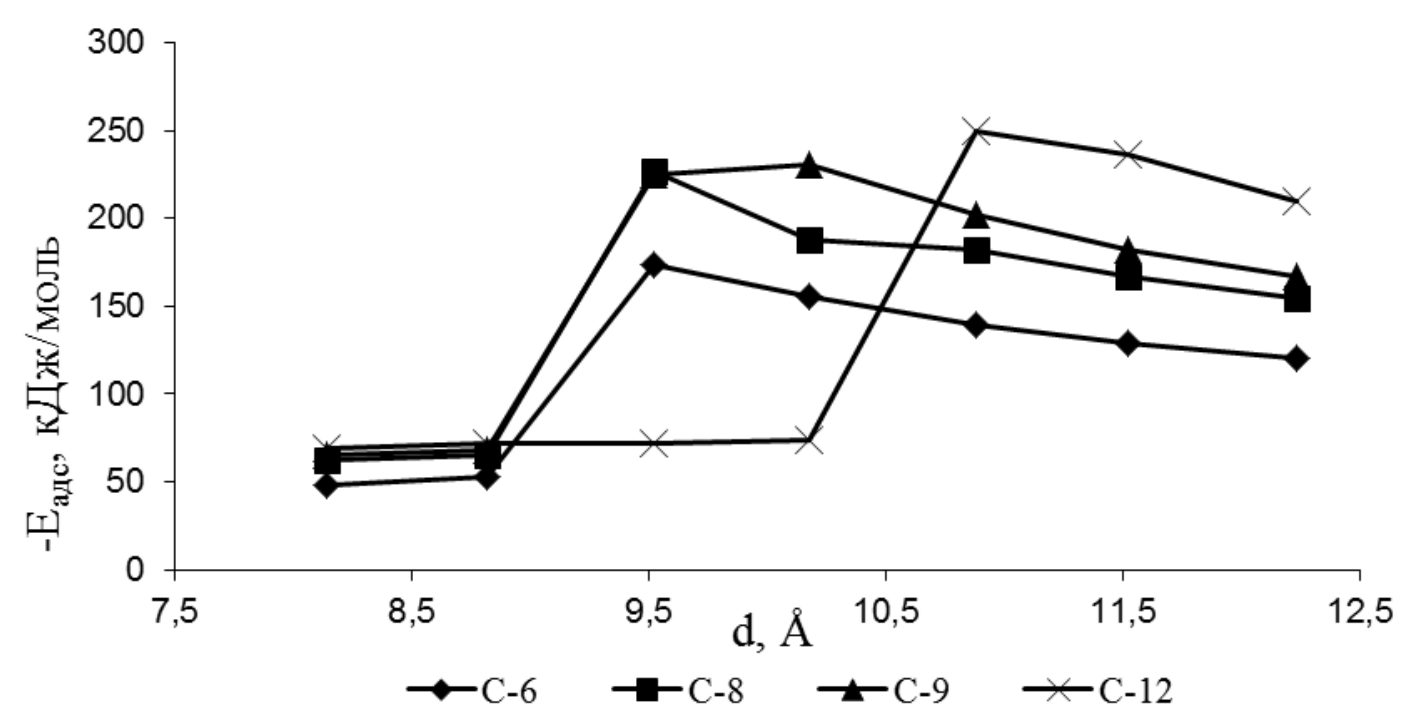

Рисунок 2. Зависимость энергии адсорбции углеводородов $\mathrm{C}_{6}$ и $\mathrm{C}_{12}$ от диаметра $\mathrm{CNT}$

Как и в случае использования полярных моделей, зависимость имеет экстремальный характер. Однако из-за симметричности канала и неполярной структуры CNT энергия адсорбции имеет максимум в области 10-11 А, что в корне отличает ее от закономерностей, наблюдаемых на полярных моделях.

Очевидно, что именно высокая полярность поверхности монтмориллонита наряду с определенным диаметром пор способствуют снижению энергии адсорбции углеводородов изостроения $\mathrm{C}_{6}-\mathrm{C}_{12}$.

В практическом плане это отражается в увеличении селективности процесса синтеза Фишера-Тропша и, как следствие, выхода целевых изоалканов.

\section{Выводы}

Таким образом, методами квантово-химического моделирования и молекулярной динамики определена оптимальная структурная характеристика (диаметр пор) для гетерогенных пористых материалов с целью интенсификации процесса синтеза Фишера-Тропша с максимальным выходом изоалканов $\mathrm{C}_{6}-\mathrm{C}_{12}$. Расчетные результаты хорошо коррелируются с экспериментальными данными, полученными в процессе 
синтеза Фишера-Тропша на разработанных катализаторах

Установлено, что при температуре $210{ }^{\circ} \mathrm{C}$ максимальный выход углеводородов изостроения $\mathrm{C}_{6}-\mathrm{C}_{12}$ составляет $78,1 \%$ масс.

Работа проведена в рамках выполнения проектной части государственного задания № 10.1448.2017/4.6.

\section{Список используемых источников}

1. Кузнецов Б.Н. Моторные топлива из альтернативного нефти сырья // Соросовский образовательный журнал. 2000. Т. 6, № 4. С. 51.

2. Пат. 2409878 РФ, МПК Н 01 М 8/06. Способ и катализатор гидрирования оксидов углерода / Кристенсен К.Х., Андерссон М., Кустов А., Йоханнессен Т., Блигаард Т., Ларсен Каспер Е., НерсковЙенс К., Зеестед Й. 2008112449/07, Заявлено 28.08.2006, Опубл. 20.01.2011. Бюл. 2.

3. Carlos Henriques. Catalysis by zeolites. EUCHEME, 2012.

4. Poursaeidesfahani A., de Lange M.F., Khodadadian F., Dubbeldam D., Rigutto M., Nair N., Vlugt Thijs J.H. Product Shape Selectivity of MFI-Type, MEL-Type, and BEAtype Zeolites in the Catalytic Hydroconversion of Heptane // J. Catal. 2017. No. 353. P. 54-62.

5. Smit B., Maesen T.L. Towards a Molecular Understanding of Shape Selectivity // Nature. 2008. No. 451. P. 671-678.

6. Demuth Thomas, Raybaud Pascal, Lacombe Sylvie, Toulhoat Hervé. Effects of Zeolite Pore Sizes on the Mechanism and Selectivity of Xylene Disproportion: a DFT Study // Journal of Catalysis. 2004. No. 222. P. 323-337.

7. Vakulin I.V., Talipov R.F., Pasko P.A., Talipova G.R., Kupova O.Yu. Features of Formation Transition States of 1,3-dioxanes by Prins Reaction in the Pores of Synthetic Zeolites A and Carbon Nanotubes // Microporous and Mesoporous Materials. 2018. No. 270. P. 30-33. 
8. Jae J., Tompsett G.A., Foster A.J., Hammond K.D., Auerbach S.M., Lobo R.F., Huber G.W. Investigation into the Shape Selectivity of Zeolite Catalysts for Biomass Conversion // J. Catal. 2011. No. 279. P. 257-268.

9. Delley B.J. An Allelectron Numerical Method for Solving the Local Density Functional for Polyatomic Molecules // Chemical Physics. 1990. No. 92. P. 508-517.

10. Pluth J.J., Smith J.V. Crystal Structure of Boggsite, a New High-Silica Zeolite with the First Three-Dimensional Channel System Bounded by both 12and 10-Rings // Am. Mineral. 1990. No. 75. P. 501-507.

11. Bu X., Feng P., Stucky G.D. Large-Cage Zeolite Structures with Multidimensional 12-Ring Channels // Science. 1997. No. 278. P. 2080-2085.

12. Каримова А.Р., Давлетшин А.Р., Рахимов М.Н., Мурзабекова А.Б. Суперкислотные катализаторы на основе кислотно-активированного монтмориллонита в синтезе Фишера-Тропша // Нефтегазохимия. 2017. № 3. C. 52-55.

\section{References}

1. Kuznetsov B.N. Motornye topliva iz al'ternativnogo nefti syr'ya [Motor fuels from alternative crude oil]. Sorosovskii obrazovatel'nyi zhurnal - Soros Educational Journal, 2000, Vol. 6, No. 4, pp. 51.

2. Kristensen K.Kh., Andersson M., Kustov A., Iokhannessen T., Bligaard T., Larsen Kasper E., NerskovIens K., Zeested I. Sposob i katalizator gidrirovaniya oksidov ugleroda [Method and Catalyst for the Hydrogenation of Carbon Oxides]. Patent RF, No. 2409878, 2011. [in Russian].

3. Carlos Henriques. Catalysis by Zeolites. EUCHEME, 2012.

4. Poursaeidesfahani A., de Lange M.F., Khodadadian F., Dubbeldam D., Rigutto M., Nair N., Vlugt Thijs J.H. Product Shape Selectivity of MFI-Type, MEL-Type, and BEAtype Zeolites in the Catalytic Hydroconversion of Heptane. J. Catal., 2017, No. 353, pp. 54-62. 
5. Smit B., Maesen T.L. Towards a Molecular Understanding of Shape Selectivity. Nature, 2008, No. 451, pp. 671-678.

6. Demuth Thomas, Raybaud Pascal, Lacombe Sylvie, Toulhoat Hervé. Effects of Zeolite Pore Sizes on the Mechanism and Selectivity of Xylene Disproportion: a DFT Study. Journal of Catalysis, 2004, No. 222, pp. 323-337.

7. Vakulin I.V., Talipov R.F., Pasko P.A., Talipova G.R., Kupova O.Yu. Features of Formation Transition States of 1,3-dioxanes by Prins Reaction in the Pores of Synthetic Zeolites A and Carbon Nanotubes. Microporous and Mesoporous Materials, 2018, No. 270, pp. 30-33.

8. Jae J., Tompsett G.A., Foster A.J., Hammond K.D., Auerbach S.M., Lobo R.F., Huber G.W. Investigation into the Shape Selectivity of Zeolite Catalysts for Biomass Conversion. J. Catal., 2011, No. 279, pp. 257-268.

9. Delley B.J. An Allelectron Numerical Method for Solving the Local Density Functional for Polyatomic Molecules. Chemical Physics, 1990, No. 92, pp. 508-517.

10. Pluth J.J., Smith J.V. Crystal Structure of Boggsite, a New High-Silica Zeolite with the First Three-Dimensional Channel System Bounded by both 12and 10-Rings. Am. Mineral., 1990, No. 75, pp. 501-507.

11. Bu X., Feng P., Stucky G.D. Large-Cage Zeolite Structures with Multidimensional 12-Ring Channels. Science, 1997, No. 278, pp. 2080-2085.

12. Karimova A.R., Davletshin A.R., Rakhimov M.N., Murzabekova A.B. Superkislotnye katalizatory na osnove kislotno-aktivirovannogo montmorillonita v sinteze Fishera-Tropsha [Superacid Catalysts Based on Acid-Activated Montmorillonite in the Fischer-Tropsch Synthesis]. Neftegazokhimiya - Oil and Gas Chemistry, 2017, No. 3, pp. 52-55. [in Russian]. 


\section{Сведения об авторах}

\section{About the authors}

Давлетшин А.Р., канд. техн. наук, доцент кафедры «Газохимия и моделирование химико-технологических процессов», ФГБОУ $\mathrm{BO}$ «УГНТУ», г. Уфа, Российская Федерация

Davletshin A.R., Candidate of Engineering Sciences, Assistant Professor of Gas Chemistry and Modeling of Chemical-Technological Processes Department, FSBEI HE «USPTU», Ufa, Russian Federation

e-mail: davletshinar@list.ru

Хамзин Ю.А., ассистент кафедры «Газохимия и моделирование химико-технологических процессов», ФГБОУ ВО «УГНТУ», г. Уфа, Российская Федерация

Khamzin Yu.A., Assistant of Gas Chemistry and Modeling of ChemicalTechnological Processes Department, FSBEI HE «USPTU», Ufa, Russian Federation

e-mail: Yunirkh@bk.ru

Шириязданов Р.Р., д-р техн. наук, доцент кафедры «Газохимия и моделирование химико-технологических процессов», ФГБОУ $\mathrm{BO}$ «УГНТУ», г. Уфа, Российская Федерация

Shiriyazdanov R.R., Doctor of Engineering Sciences, Assistant Professor of Gas Chemistry and Modeling of Chemical-Technological Processes Department, FSBEI HE «USPTU», Ufa, Russian Federation

e-mail: petroleum9@bk.ru

Пасько П.А., аспирант кафедры «Органическая и биоорганическая химия», ФГБОУ ВО «БашГУ», г. Уфа, Российская Федерация

Pasko P.A., Post-Graduate Student of Organic and Bioorganic Chemistry Department, FSBEI HE «BashSU», Ufa, Russian Federation

e-mail: pasko.pav62@gmail.com 
Вакулин И.В., д-р хим. наук, профессор кафедры «Органическая и биоорганическая химия», ФГБОУ ВО «БашГУ», г. Уфа, Российская Федерация

Vakulin I.V., Doctor of Chemical Sciences, Professor of Organic and Bioorganic Chemistry Department, FSBEI HE «BashSU», Ufa, Russian Federation

$$
\text { e-mail: vakuliniv@mail.ru }
$$

Талипов Р.Ф., д-р хим. наук, профессор кафедры «Органическая и биоорганическая химия», ФГБОУ ВО «БашГУ», г. Уфа, Российская Федерация

Talipov R.F., Doctor of Chemical Sciences, Professor of Organic and Bioorganic Chemistry Department, FSBEI HE «BashSU», Ufa, Russian Federation 